\title{
Circular RNA circCSPP1 promotes the occurrence and development of colon cancer by sponging miR-431 and regulating ROCK1 and ZEB1
}

Jin Wang ${ }^{1,2,3,4,5+}$, Lei Zhou ${ }^{6 \dagger}$, Bingxin Chen ${ }^{7 \dagger}$, Zhuwen $\mathrm{Yu}^{7 \dagger}$, Jianglei Zhang ${ }^{8}$, Zhe Zhang ${ }^{1,2}$, Chenrui Hu', Yanjin Bai ${ }^{1}$, Xiaokang Ruan ${ }^{1}$, Shengjia Wang ${ }^{1}$, Jun Ouyang ${ }^{8}$, Airong Wu ${ }^{7 *}$ and Xin Zhao ${ }^{1,3,4,5^{*}}$

\begin{abstract}
Background: Colon cancer is a common malignant tumor of the digestive tract, and its incidence is ranked third among gastrointestinal tumors. The present study aims to investigate the role of a novel circular RNA (circCSPP1) in colon cancer and its underlying molecular mechanisms.

Methods: Bioinformatics analysis and reverse transcription-quantitative PCR were used to detect the expression levels of circCSPP1 in colon cancer tissues and cell lines. The effects of circCSPP1 on the behavior of colon cancer cells were investigated using CCK-8, transwell and clonogenic assays. Bioinformatics analysis along with luciferase, fluorescence in situ hybridization and RNA pull-down assays were used to reveal the interaction between circCSPP1, microRNA (miR)-431, Rho associated coiled-coil containing protein kinase 1 (ROCK1) and zinc finger E-box binding homeobox 1 (ZEB1).

Results: It was found that circCSPP1 expression was significantly upregulated in colon cancer tissues and cell lines. Overexpression of circCSPP1 significantly promoted the proliferation, migration and invasion of colon cancer cells, whereas silencing of circCSPP1 exerted opposite effects. Mechanistically, circCSPP1 was found to bind with miR-431. In addition, ROCK1 and ZEB1 were identified as the target genes of miR-431. Rescue experiments further confirmed the interaction between circCSPP1, miR-431, ROCK1 and ZEB1. Moreover, circCSPP1 promoted the expression level of ROCK1, cyclin D1, cyclin-dependent kinase 4, ZEB1 and Snail, and lowered the E-cadherin expression level.
\end{abstract}

Conclusion: Taken together, the findings of the present study indicated that circCSPP1 may function as a competing endogenous RNA in the progression of colon cancer by regulating the miR-431/ROCK1 and miR-431/ZEB1 signaling axes.

Keywords: circCSPP1, miR-431, Rho associated coiled-coil containing protein kinase 1, Zinc finger E-box binding homeobox 1, Colon cancer, Cell cycle, Epithelial-to-mesenchymal transition

*Correspondence: arwu@suda.edu.cn; zhaox@suda.edu.cn

†Jin Wang, Lei Zhou, Bingxin Chen and Zhuwen Yu contributed equally to this work

${ }^{1}$ Department of General Surgery, The First Affiliated Hospital of Soochow University, Suzhou, China

${ }^{7}$ Department of Gastroenterology, The First Affiliated Hospital of Soochow University, Suzhou, China

Full list of author information is available at the end of the article

\section{Background}

Colon cancer is a common malignant tumor of the digestive tract, and its incidence is ranked third among gastrointestinal tumors [1]. Over the past few decades, the rapid development of molecular biology has enriched the theory of colorectal cancer carcinogenesis [1-4]. In 
addition, immense progress has been made in diagnostic and treatment strategies for colorectal cancer; the 5 -year survival rate of patients with localized disease is 90.1\% [5]. However, following the metastasis of colorectal cancer to adjacent organs or lymph nodes, the 5-year survival rate of patients decreases to $69.2 \%$. Of note, only $39 \%$ of patients with colorectal cancer are diagnosed at the localized stage of the disease, prior to metastasis $[6,7]$. Therefore, further in-depth investigations of the pathogenesis of colorectal cancer, as well as the identification of more effective early diagnosis and treatment strategies are essential for colorectal cancer.

MicroRNAs (miRNAs/miRs) are small endogenous single-stranded RNA molecules composed of $\sim 20$ nucleotides, which act mostly on the $3^{\prime}$ UTR of target mRNAs and either degrade or inhibit multiple transcripts $[8,9]$. Previous studies have demonstrated that miRNAs play a critical regulatory role in the initiation and progression of human cancers [10,11].

Circular RNAs (circRNAs) are newly discovered noncoding RNAs with a covalently closed ring structure, which are widely found in a variety of cells [12-15]. They are produced by the reverse splicing of precursor mRNAs and characterized by a stable structure, a conserved sequence and tissue specificity. Recent studies have indicated that circRNAs can act as miRNAs sponges to inhibit the activity of targeted miRNAs [16, 17]. In addition, circRNAs can regulate gene transcription by binding with RNA binding proteins, or can be translated to produce proteins [18]. Thus, circRNAs play a vital role during the progression of tumors, and may provide a novel direction for tumor diagnosis and therapy $[19,20]$.

In the present study, the commonly differentially expressed circRNAs between colon cancer tissues and adjacent normal tissues in two public datasets were screened out to identify novel molecular targets for colon cancer treatment. It was found that circCSPP1 was significantly upregulated in cancer tissues. In addition, the role of circCSPP1 in colon cancer was examined in vitro and in vivo.

\section{Materials and methods}

\section{Specimen collection}

Cancer tissues and adjacent normal tissues were collected from 25 patients (14 male, 11 female), who diagnosed with colon cancer at the First Affiliated Hospital of Soochow University (Suzhou, China) (August, 2020 to July, 2021). These patients received no treatment before and age of them was range from 37 to 72 -year-old. The tissues were stored in liquid nitrogen immediately after resection. The present study was approved by the Ethics Committee of the First Affiliated Hospital of Soochow
University (No. FAHSU20200719) and written informed consent was obtained from each patient.

\section{Gene expression omnibus (GEO) data analysis}

The present study analyzed the GSE121895 and GSE126094 datasets from the GEO database. The expression levels in each group were normalized. The threshold value of differentially expressed genes was set at two of different multiples and $\mathrm{p}<0.05$.

\section{Cell culture and transfection}

The human colonic epithelial cell line (HFC) was purchased from ScienCell Research Laboratories, Inc. Colon cancer cell lines, including SW620, SW480, LOVO, HCT116 and DLD-1 cells were obtained from the American Type Culture Collection (ATCC). THP-1 cells were also obtained from ATCC. The cells were maintained in DMEM (Thermo Fisher Scientific, Inc.) containing 10\% FBS supplemented with $100 \mathrm{U} / \mathrm{ml}$ penicillin and $100 \mathrm{~g} /$ $\mathrm{ml}$ streptomycin (Beyotime Institute of Biotechnology) at $37^{\circ} \mathrm{C}$. When the cell density (SW620, LOVO) reached $50-70 \%$, the cells were transfected with miR-431 mimics (20 nM), mimics control, circCSPP1 pcDNA3.1 overexpression plasmid $(1 \mu \mathrm{g} / \mu \mathrm{l})$ or circCSPP1 pLVX-IRES-Puro silencing plasmid (shRNA1 and shRNA2; $1 \mu \mathrm{g} / \mu \mathrm{l}$ ) for 24 h using Lipofectamine ${ }^{\circledR} 3000$ (Thermo Fisher Scientific, Inc.) according to the manufacturer's instructions. The miR-431 mimics, miR-control, circCSPP1 pcDNA3.1 overexpression plasmid, circCSPP1 pLVX-IRES-Puro silencing plasmids; Rho associated coiled-coil containing protein kinase 1 (ROCK1) and ZEB1 pLVX-IRES-Puro silencing plasmids were obtained from Shanghai Genepharma Co., Ltd. Phorbol-12-myristate-13-acetate (PMA), IL-4 and IL-13 were purchased from Sigma-Aldrich; Merck KGaA. The information of oligonucleotide was provided in Table 1.

\section{Reverse transcription-quantitative PCR (RT-qPCR)}

TRIzol $^{\circledR}$ reagent (Thermo Fisher Scientific, Inc.) was used to extract the RNA according to the manufacturer's protocol. The RNA was reverse transcribed into cDNA using a reverse transcription kit (Takara Bio, Inc.). The expression levels of miR-431, ROCK1 and zinc finger E-box binding homeobox 1 (ZEB1) were detected using a fluorescence quantitative PCR kit (Nanjing Jiancheng Bioengineering Inc.) in a BD FACSVerse ${ }^{\mathrm{TM}}$ (BD Biosciences). U6 and GAPDH were used as internal controls for miR431 and mRNAs, respectively. Real-Time qPCRs were used three times: 2 min at $94{ }^{\circ} \mathrm{C}$, followed by 35 cycles $\left(94{ }^{\circ} \mathrm{C}\right.$ for $30 \mathrm{~s}$ and $55^{\circ} \mathrm{C}$ for $45 \mathrm{~s}$ ). The primers used were as follows: RT primer for miR-431, 5'-GTCGTATCC AGTGCAGGGTCCGAGGTGCACTGGATACGACAC GUACU-3'; miR-431 forward, 5'-TGCGGUGUCUUG 
Table 1 The information of oligonucleotide sequences

\begin{tabular}{|c|c|}
\hline Gene & Sequence $\left(5^{\prime}-3^{\prime}\right)$ \\
\hline ROCK1 shRNA & $\begin{array}{l}\text { sense: CACCGCATTTGGAGAAGTTCAATTGCGAACAATTGAACTTCTCCAAATGC } \\
\text { antisense: AAAAGCATTTGGAGAAGTTCAATTGTTCGCAATTGAACTTCTCCAAATG }\end{array}$ \\
\hline \multirow[t]{2}{*}{ ZEB1 shRNA } & sense: CACCGAGAGAGAGAGTTTGACAAGGCGAACCTTGTCAAACTCTCTCTCTC \\
\hline & antisense: AAAAGAGAGAGAGAGTTTGACAAGGTTCGCCTTGTCAAACTCTCTCTCTC \\
\hline circCSPP1 shRNA1 & $\begin{array}{l}\text { sense: CACCGCTCCAGACAATGAAACATCCCGAAGGATGTTTCATTGTCTGGAGC } \\
\text { antisense: AAAAGCTCCAGACAATGAAACATCCTTCGGGATGTTTCATTGTCTGGAGC }\end{array}$ \\
\hline circCSPP1 shRNA2 & $\begin{array}{l}\text { sense: CACCGCTAATCAAGATACCTGTAGTCGAAACTACAGGTATCTTGATTAGC } \\
\text { antisense: AAAAGCTAATCAAGATACCTGTAGTTTCGACTACAGGTATCTTGATTAGC }\end{array}$ \\
\hline shRNA control & $\begin{array}{l}\text { sense: GATCCGTTCTCCGAACGTGTCACGTTTCAAGAGAACGTGACACGTTCGGAG } \\
\text { AACTTTTTTG } \\
\text { antisense: AATTCAAAAAAGTTCTCCGAACGTGTCACGTTCTCTTGAAACGTGACA } \\
\text { CGTTCGGAGAACG }\end{array}$ \\
\hline miR-431 mimic & UGUCUUGCAGGCCGUCAUGCA \\
\hline mimic control & UUCUCCGAACGUGUCACGUTT \\
\hline miR-431 inhibitor & UGCAUGACGGCCUGCAAGACA \\
\hline inhibitor control & UUCUCCGAACGUGUCACGUTT \\
\hline miR-324-5p mimic & CGCAUCCCCUAGGGCAUUGGUG \\
\hline miR-375 mimic & UUUGUUCGUUCGGCUCGCGUGA \\
\hline miR-486-3p mimic & CGGGGCAGCUCAGUACAGGAU \\
\hline
\end{tabular}

CAGGCCGUCAG-3' and reverse, 5'-CCAGTGCAG GGTCCGAGGT-3'; U6 forward, 5'-CTCGCTTCGGCA GCACA- $3^{\prime}$ and reverse, $5^{\prime}$-AACGCTTCACGAATTTGC GT-3'; ROCK1 forward, 5'-AACATGCTGCTGGATAAA TCTGG-3' and reverse, 5'-TGTATCACATCGTACCAT GCCT-3'; ZEB1 forward, 5'-TTCTCACACTCTGGG TCTTATTCTC-3' ${ }^{\prime}$ and reverse, 5'-CTTTTTCACTGT CTTCATCCTCTTC-3'; arginase-1 forward, 5'-AGA CCACAGTTTGGCAATTGG- $3^{\prime}$ and reverse, 5'-AGG AGAATCCTGGCACATCG-3'; IL-10 forward, 5'-AAC CTGCCTAACATGCTTCG-3' and reverse, $5^{\prime}$-GAGTTC ACATGCGCCTTGAT-3'; circCSPP1 forward, 5'-CCA TCCCATCAGTTCATCCT- $3^{\prime}$ and reverse, $5^{\prime}$-CCCTGC AAAAGGACTACAGG-3'; SMAD4 forward, 5'-GCT GCTGGAATTGGTGTTGA-3' and reverse, $5^{\prime}$-CTTCGT CTAGGAGCTGGAGG-3'; DAAM1 forward, 5'-TTC ATTCATCTTTTGCTGTTTCCGA-3 ${ }^{\prime}$ and reverse, 5'-TTTTCTTCCTGGTCCTTTTTCTTGC-3'; CDK14 forward, 5'-GCACAGAGACCTGAAACCACAG-3' and reverse, 5'-AAAGATGCAACCTACTCCCCAC3'; GAPDH forward, 5'-TCAAGAAGGTGGTGAAGC AGG-3' and reverse, 5'-TCAAAGGTGGAGGAGTGG GT-3'. The data were quantified by using $2^{-\Delta \Delta t}$ method [21]. All these experiments were performed in triplicate.

\section{Cell counting kit-8 (CCK-8) assay}

The SW620 or LOVO cells $\left(3 \times 10^{5}\right)$ were seeded in 96-well plates and cultured for 0, 24, 48 and $72 \mathrm{~h}$. At each time point, the cells were incubated with $10 \mu \mathrm{l} \mathrm{CCK-8}$ solution (Beyotime) at $37{ }^{\circ} \mathrm{C}$ for $4 \mathrm{~h}$. The optical density was then measured at $450 \mathrm{~nm}$ as previously described [22]. All these experiments were performed in triplicate.

\section{Cell colony forming assay}

The cells $\left(5 \times 10^{3}\right)$ were suspended in DMEM containing $10 \% \mathrm{FBS}$, and then seeded into the plate. After 2 weeks of incubation at $37{ }^{\circ} \mathrm{C}$, the cells were fixed with $5 \mathrm{ml} 4 \%$ paraformaldehyde for $15 \mathrm{~min}$. The cells were then stained with Giemsa (Beyotime) for $30 \mathrm{~min}$. The number of colonies was counted using a light microscope $(200 \times$; Nikon Corporation). All these experiments were performed in triplicate.

\section{Transwell assay}

The cells $\left(2 \times 10^{4}\right)$ were digested and cultured in a serumfree medium in the Transwell (BD) upper chamber with or without Matrigel (BD Biosciences). Subsequently, $600 \mu \mathrm{l}$ complete medium ( $10 \%$ serum) were added to the lower chamber. After $24 \mathrm{~h}$ of incubation at $37^{\circ} \mathrm{C}$, the cells in the lower chamber were fixed with $4 \%$ formaldehyde for $10 \mathrm{~min}$ at room temperature and stained with $0.1 \%$ crystal violet solution at room temperature for $10 \mathrm{~min}$ (Sigma-Aldrich; Merck KGaA). Finally, the migrated or invaded cells were photographed using a light microscope $(200 \times)$ [23]. All these experiments were performed in triplicate.

\section{Fluorescence in situ hybridization (FISH) assay}

Cy3-labeled circCSPP1 and FITC-labeled miR-431 probes (Biosense Technologies) were used to observe the 
co-localization of circCSPP1 and miR-431 in the cells. Hybridizations were performed according to the manufacturer's instructions provided with the fluorescence in situ hybridization kit. The cell nuclei were stained with DAPI at room temperature for $20 \mathrm{~min}$. Subsequently, images were visualized using a fluorescence microscope $(200 \times)$ as previously described [24].

\section{Luciferase assay}

The luciferase assay was performed using the dual-luciferase reporting system psiCHECK (Thermo Fisher Scientific, Inc.). The wild-type (WT) or mutant-type (mut) sequences of circCSPP1, ROCK1 and ZEB1 were cloned into the psiCHECK2 plasmid. $293 \mathrm{~T}$ cells (ATCC, $2 \times 10^{4}$ cells/well) were cultured overnight in 24 -well plates. The cells were transfected with the WT or mut reporter vector along with miR-431 mimics $(10 \mathrm{nM})$ or mimics control (10 nM) using Lipofectamine ${ }^{\circledR} 3000$ (Thermo Fisher Scientific, Inc.). Finally, the luciferase activity of cells was detected with a Dual-Luciferase Detection kit (Promega Corporation) after $48 \mathrm{~h}$ of transfection. The data were quantified by normalizing to Renilla luciferase activity.

\section{RNA pull-down assay}

Biotin labeled miR-431 and the control probes were synthesized by Sangon Biotech (Shanghai) Co., Ltd. Probecoated beads were generated by co-incubation with streptavidin-coated beads (Thermo Fisher Scientific, Inc.) at $25{ }^{\circ} \mathrm{C}$ for $2 \mathrm{~h}$. The SW620 and LOVO cells were collected, lysed and incubated with miR-431 probes overnight at $4{ }^{\circ} \mathrm{C}$. Thereafter, the beads were eluted, and the complex was purified using TRIzol ${ }^{\circledR}$ reagent (Takara Biotechnology Co., Ltd.). The levels of circCSPP1, ROCK1 and ZEB1 were then analyzed using RT-qPCR.

\section{RNA immunoprecipitation (RIP) assay}

RIP assay was performed using the EZ-Magna RIP RNA-Binding Protein Immunoprecipitation kit (MilliporeSigma). Briefly, magnetic beads conjugated with negative control normal IgG (cat.no. AB21-KC, 1:5000) or anti-Ago2 (cat.no. 03-110, 1:5000) antibody (MilliporeSigma) were co-incubated with the cell lysates for $4 \mathrm{~h}$ at room temperature. To investigate the enrichment of the binding targets, the immunoprecipitated RNAs were extracted and subjected to RT-qPCR.

\section{Western blot analysis}

RIPA lysis buffer (Beyotime Institute of Biotechnology) was used to extract protein from the cells. The protein concentration was determined using the BCA kit (Nanjing Jiancheng Bioengineering Inc.) according to the manufacturer's instructions. Protein $(40 \mu \mathrm{g})$ was then separated by using $10 \%$ SDS-PAGE, and transferred onto
PVDF membranes (MilliporeSigma). The membranes were blocked in 5\% skimmed milk for $1 \mathrm{~h}$ at room temperature followed by incubation with the following primary antibodies: ROCK1 (cat. no. \#4035, 1:1,000, Cell Signaling Technology, Inc.), ZEB1 (cat.no. ab181451, 1:1,000, Abcam), cyclin D1 (cat. no. ab16663, 1:1,000, Abcam), cyclin-dependent kinase (CDK)4 (cat.no. 110261-AP, 1:1,000, ProteinTech Group, Inc.), p-CDK4 (1:1,000, Abcam), retinoblastoma (Rb; cat. no. ab181616, 1:1,000, Abcam), p-Rb (cat.no. ab184796, 1:1,000, Abcam), Snail (cat.no. ab216347, 1:1,000, Abcam), E-cadherin (E-cad; cat. no. 20874-1-AP, 1:1,000, ProteinTech Group, Inc.) and GAPDH (1:1500, cat. no. HRP-60004, ProteinTech Group, Inc.) at $4{ }^{\circ} \mathrm{C}$ overnight. The membranes were then incubated with HRP-labeled goat anti-rabbit secondary antibody (Abcam, cat. no. ab7090; 1:5,000) at room temperature for $1 \mathrm{~h}$. Thereafter, an enhanced chemiluminescence kit (Thermo Fisher Scientific, Inc.) was used to detect protein expression. All these experiments were performed in triplicate.

\section{Xenograft tumor model}

Nude mice ( $\mathrm{n}=24,4-6$ weeks old, 20-22 g) were obtained from the Animal center of Soochow University and randomly divided into four groups (shRNA2 ctrl, circCSPP1 shRNA2, pcDNA3.1 ctrl and pcDNA3.1circCSPP1). All mice were housed in a SPF-grade animal room (temperature $18-22{ }^{\circ} \mathrm{C}$; humidity $40-60 \%$; light/ dark cycle $12 / 12 \mathrm{~h}$ each day) and had free access to food and water. The subcutaneous injection of colon cancer cells was performed after 3 days of adaptive breeding. Each mouse was subcutaneously injected with $3 \times 10^{6}$ colon cancer cells $(100 \mu \mathrm{l}$ in PBS). Tumor size was measured every 2 days, and the major axis (a) and minor axis (b) of the tumor were measured. The tumor volume was calculated using the following formula: $\mathrm{ab}^{2} / 2$. At the end of the experiment, the mice were sacrificed using a $40 \%$ volume/min $\mathrm{CO}_{2}$ and the tumors were removed, photographed and weighed. The animal experiments were approved by the Ethics Committee of the First Affiliated Hospital of Soochow University (Approval No. 20200917). The National Institutes of Health guide for the care and use of laboratory animals was strictly followed.

\section{Cell cycle distribution analysis}

SW620 or LOVO cells $\left(5 \times 10^{5}\right)$ were fixed using with $75 \%$ ethanol for $20 \mathrm{~min}$ on ice. Then, cells were permeabilized with $0.25 \%$ Triton X-100 and stained with PI/RNase (Sigma Aldrich). After $15 \mathrm{~min}$ of incubation at $4{ }^{\circ} \mathrm{C}$, cells were analyzed using a flow cytometer (BD FACSAria III; BD Biosciences) and ModFit (version 3.0; Verity Software House, Inc.). All these experiments were performed in triplicate. 


\section{Statistical analysis}

Three independent experiments were performed in each group. Statistical analysis was performed using GraphPad Prism software (GraphPad Software, Inc.). The measurement data are expressed as the mean \pm standard deviation. The unpaired Student's t-test was used for comparisons between two groups, and One-way analysis of variance and Tukey's post hoc tests were used for comparisons between multiple groups [25]. $\mathrm{p}<0.05$ was considered to indicate a statistically significant difference.

\section{Results}

\section{CircCSPP1 is highly expressed in colon cancer}

To explore novel molecular targets for the treatment of colon cancer, the differentially expressed circRNAs between cancer and adjacent normal tissues were first analyzed using two GEO datasets (GSE121895 and GSE126094) (Fig. 1A). A total of 161 differentially expressed circRNAs were identified by intersection analysis of the two transcriptomics data (Fig. 1B). Further RT-PCR verification at the tissue level revealed that hsa circ_0001806 (circCSPP1) was significantly upregulated in colon cancer (Fig. 1C and D). Consistently, compared with the HFC cells, the expression of circCSPP1 was found to be upregulated in colon cancer cells (Fig. 1E). In addition, circRNA circularization data indicated that circCSPP1 was spliced by exons $8-11$ of the CSPP1 transcript, which was confirmed by Sanger sequencing (Fig. 1F).

Subsequently, the distribution of circCSPP1 in the cells was detected using FISH assay and RT-qPCR. The data revealed that circCSPP1 was mainly located in the cytoplasm (Fig. 1G and H). Compared with linear RNA, circCSPP1 was more resistant to actinomycin or RNase $\mathrm{R}$ treatment (Fig. 1I and J). These data thus indicated that circCSPP1 had higher stability and a longer half-life.

\section{Knockdown of circCSPP1 significantly inhibits the tumorigenesis of colon cancer}

In order to investigate the function of circCSPP1 in colon cancer, cell proliferation, invasion and migration were detected. First, circCSPP1 was knocked down in colon cancer cells using shRNA1 and shRNA2. The results of RT-qPCR revealed that both these shRNAs effectively suppressed the level of circCSPP1 in the cells (Fig. 2A). In addition, the results of the CCK- 8 assay demonstrated that knockdown of circCSPP1 significantly inhibited the proliferation of colon cancer cells (Fig. 2B). Consistently, knockdown of circCSPP1 notably decreased the colony-forming ability of the cells (Fig. 2C). Moreover, transwell assay revealed that knockdown of circCSPP1 inhibited the invasive and migratory ability of colon cancer cells (Fig. 2D and E). These data thus suggested that knockdown of circCSPP1 significantly inhibited the progression of colon cancer in vitro.

\section{CircCSPP1 promotes colon cancer tumor growth and metastasis in vivo}

With the purpose of confirming the biological function of circCSPP1 in colon cancer, an animal experiment was performed. The results of the animal experiment revealed that the overexpression of circCSPP1 significantly promoted tumor growth, whereas the knockdown of circCSPP1 inhibited tumor growth (Fig. 3A-D). Moreover, the metastasis of colon cancer in vivo was also assessed. The results showed that knockdown of circCSPP1 significantly reduced the metastasis of colon cancer, while overexpression of circCSPP1 promoted the metastasis of colon cancer (Fig. 3E and F). In addition, the expression level of circCSPP1 in tumor tissues was inhibited by circCSPP1 shRNA2 (Fig. 3G). On the whole, these indicated that circCSPP1 promoted colon cancer tumor growth and metastasis in vivo.

\section{CircCSPP1 sponges with miR-431 in colon cancer cells}

Circinteractome database (https://circinteractome. irp.nia.nih.gov/) was used to explore the potential target of circCSPP1. A total of five miRNAs (miR-197, miR-324-5p, miR-375, miR-431 and miR-486-3p) were predicted to be the candidate targets of circCSPP1 (Additional file 1: Fig. S1A). Luciferase reporter assay was then used to screen the candidate binding miRNAs. The results revealed that the relative luciferase activities of the cells were most notably inhibited by miR- $431 \mathrm{mim}$ ics (Additional file 1: Fig. S1A). The binding site between circCSPP1 and miR-431 is presented in Additional file 1: Fig. S1B. Subsequently, luciferase reporter assay confirmed that circCSPP1 was able to bind to miR-431 in SW620 and LOVO cells (Additional file 1: Fig. S1C). In addition, the results of FISH assay revealed the co-localization of circCSPP1 with miR-431 in the cytoplasm of the cells (Additional file 1: Fig. S1D). Moreover, RIP and RNA pull-down assays revealed that circCSPP1 could directly bind with miR-431 (Additional file 1: Fig. S1Eand F). Thus, these data suggested that circCSPP1 sponged miR-431 in colon cancer cells.

\section{ROCK 1 is a target gene of miR-431 in colon cancer cells}

Then, TargetScan (http://www.targetscan.org/vert_72/), miRDB (http://www.mirdb.org/) and miRWalk (http:// zmf.umm.uni-heidelberg.de/apps/zmf/mirwalk/micro rnapredictedtarget.html) were used to explore the target genes of miR-431 in colon cancer cells. The expression of several potential targets involved in cancer development was assessed. Based on these three databases, ZEB1, SMAD4, disheveled associated activator of 


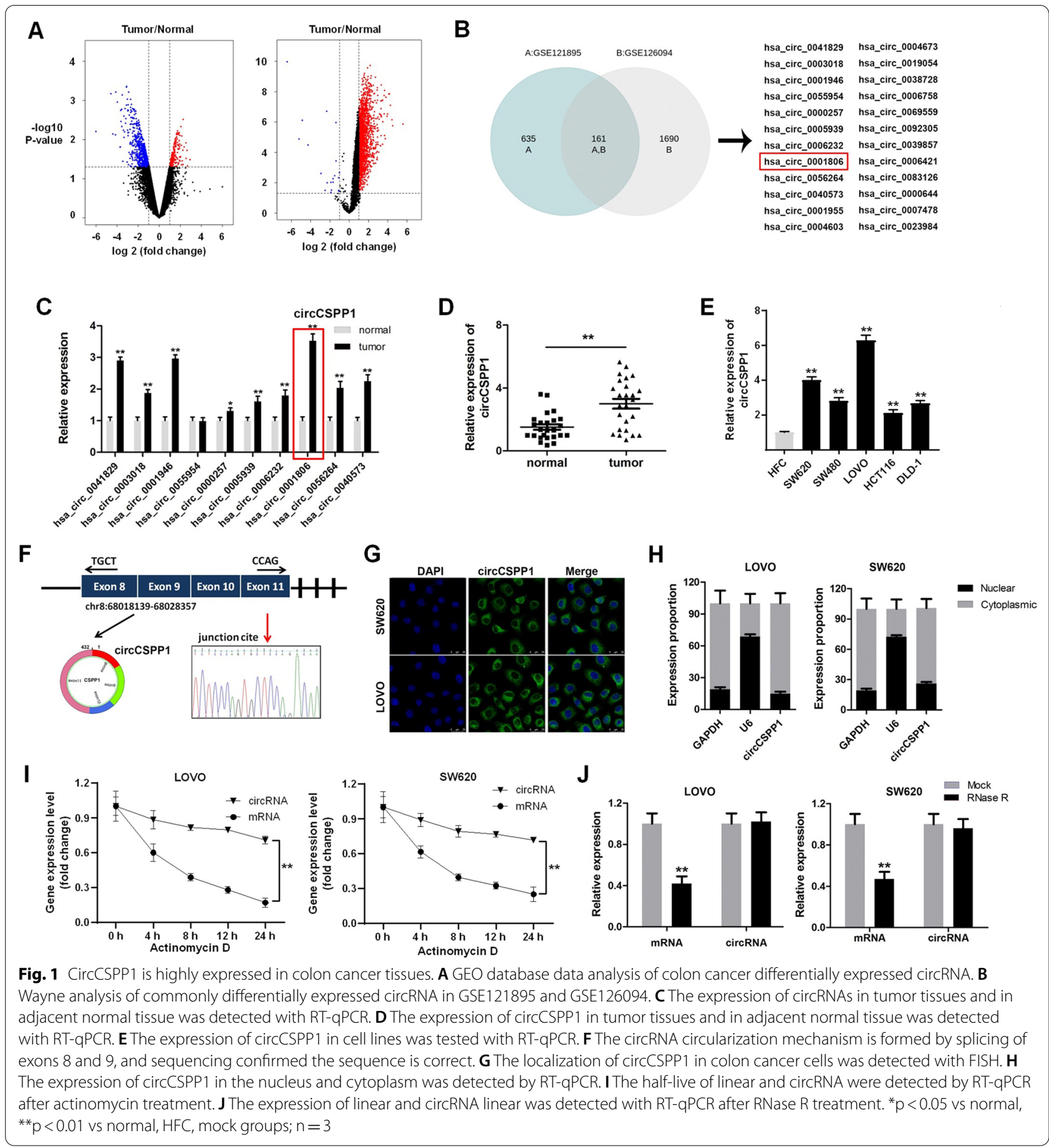

morphogenesis 1 (DAAM1), CDK14 and ROCK1 were predicted to be the candidate targets of miR-431 (Additional file 2: Fig. S2A). ZEB1 and ROCK1 were found to be downregulated by miR-431 mimics (Additional file 2: Fig. S2A). Among these genes, ROCK1 was first selected for further analysis due to its critical role in the progression of cancer [26].
RT-qPCR results revealed that ROCK1 expression was notably upregulated in colon cancer tissues compared with adjacent normal tissues (Additional file 2: Fig. S2B). The potential complementary pairing sequence between miR-431 and the 3'-UTR of ROCK1 is presented in Additional file 2: Fig. S2C. In addition, luciferase reporter assay indicated that the luciferase activity of the cells 


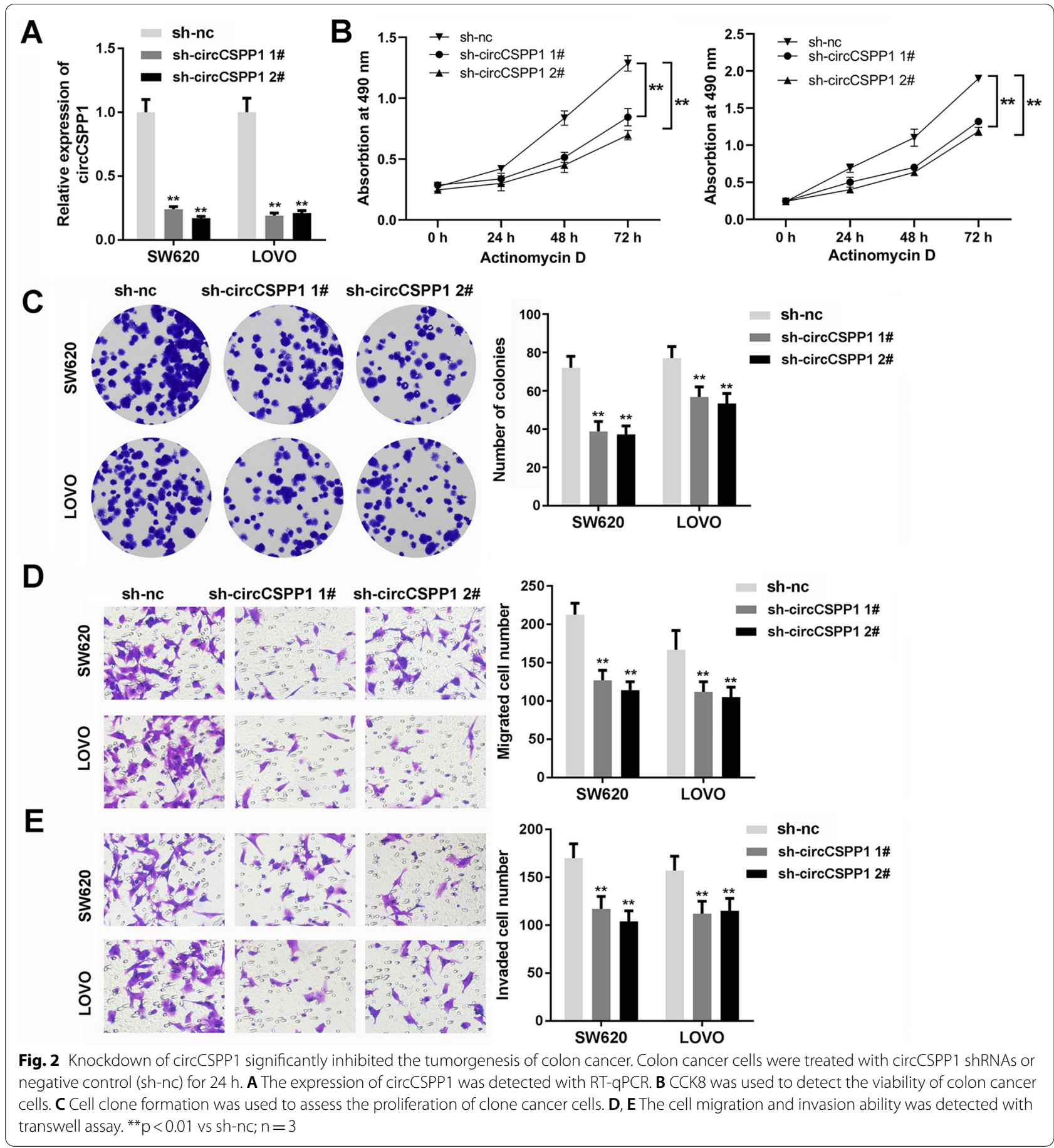

carrying the WT ROCK1 $3^{\prime}$-UTR was significantly reduced by miR-431 mimics (Additional file 2: Fig. S2D). Consistently, the results of RIP and RNA pull-down assays revealed the direct interaction between miR-431 and ROCK1 (Additional file 2: Fig. S2E and F). On the whole, these data confirmed that ROCK1 was a target gene of miR-431 in colon cancer cells.

\section{Knockdown of ROCK1 reverses the tumor-promoting} effects of circCSPP1

To further confirm the interaction among circCSPP1, miR-431 and ROCK1, rescue experiments were performed. The results of RT-qPCR revealed that the overexpression of circCSPP1 promoted ROCK1 expression, whereas this effect was reversed by transfection with 


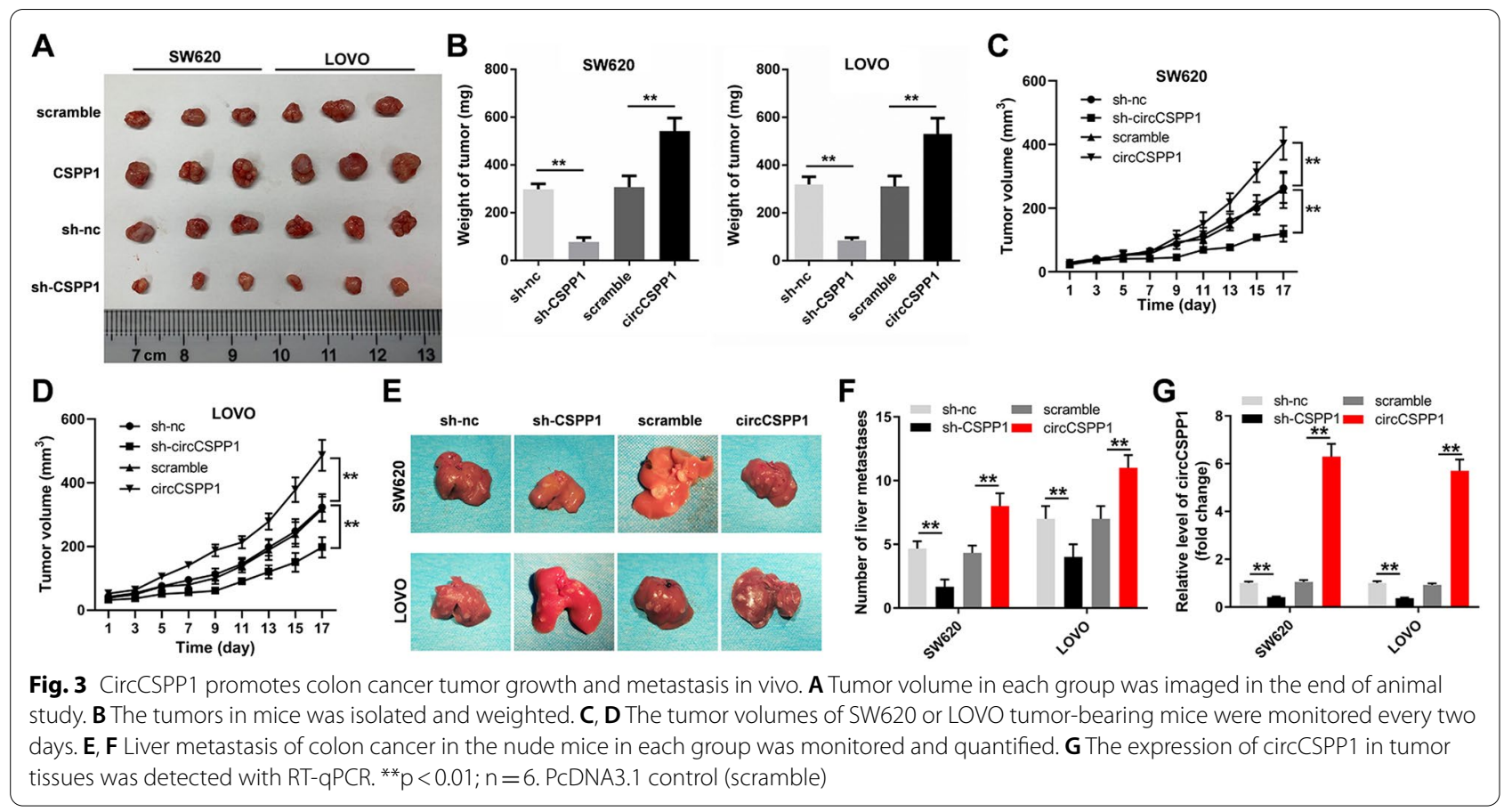

miR-431 mimics (Fig. 4A). In addition, the results of CCK-8 and colony formation assays indicated that circCSPP1 significantly increased the proliferation of colon cancer cells, which was reversed by transfection with miR-431 mimics or ROCK1 knockdown (Fig. 4B and C). Consistently, the transwell assay results revealed that circCSPP1 notably promoted the migration and invasion of colon cancer cells, whereas these effects were reversed by miR-431 mimics or ROCK1 knockdown (Fig. 4D and E). Additionally, the effects of circCSPP1, sh-ROCK1 or sh-ZEB1 on their target genes in cells were detected with RT-qPCR, respectively (Additional file 4: Fig. S4A-C). Meanwhile, miR-431 mimics significantly increased the level of miR-431, while miR-431 inhibitor exhibited completely opposite effect (Additional file 4: Fig. S4D and F). Taken together, these findings demonstrated that the knockdown of ROCK1 reversed the tumor-promoting effects of circCSPP1.

\section{MiR-431 targets ZEB1 in colon cancer cells}

Then we explored the interaction among circCSPP1, miR-431 and ZEB1. The potential complementary pairing sequence between miR-431 and the $3^{\prime}$-UTR of ZEB1 is presented in Fig. 5A. The results of luciferase reporter experiment indicated that miR-431 mimics significantly reduced the luciferase activity of cells carrying the WT ZEB1 3'-UTR (Fig. 5B). In addition, RT-qPCR and western blot analyses revealed that miR-431 mimics notably decreased ZEB1 expression at the mRNA and protein level in the cells; by contrast, miR-431 inhibitor increased ZEB1 expression (Fig. 5C and D). Moreover, RIP assay using the antibody against Ago2 confirmed the interaction between miR-431 and ZEB1 (Fig. 5E).

Subsequently, the interaction between circCSPP1 and ZEB1 was investigated using RT-qPCR. The results revealed that the overexpression of circCSPP1 promoted the expression of ZEB1. However, this phenomenon was completely reversed by transfection with miR-431 mimics or ZEB1 knockdown (Fig. 5F). Similarly, the promoting effects of circCSPP1 on cell invasion and migration were significantly inhibited by ZEB1 knockdown (Fig. 5G and $\mathrm{H})$. Thus, these data illustrated miR-431 targeted ZEB1 in colon cancer cells.

\section{CircCSPP1 activates the cyclin D1/CDK4/Rb signaling pathway in colon cancer}

To further examine the role of the circCSPP1 in colon cancer, the levels of cell cycle-related proteins were detected using western blot analysis. The results indicated that the overexpression of circCSPP1 increased the expression of ROCK1, cyclin D1, p-CDK4 and p-Rb in the cells. However, these phenomena were reversed by transfection with miR-431 mimics or by ROCK1 knockdown (Fig. 6A). Moreover, it was found that circCSPP1 overexpression also increased the expression of ZEB1 and Snail, and downregulated the E-cadherin level. Similarly, the effects of circCSPP1 overexpression on these proteins were reversed by transfection with miR-431 mimics or 


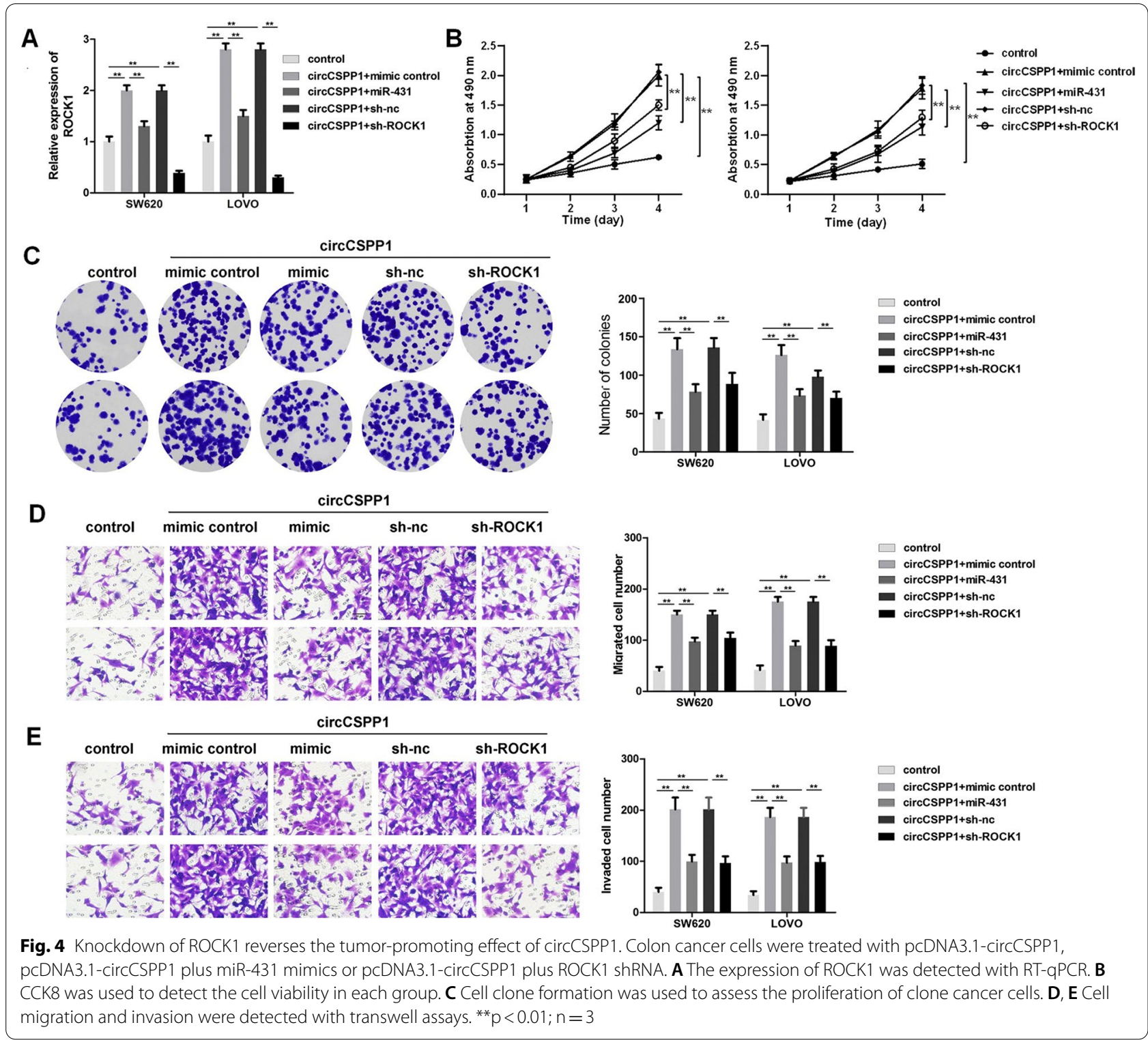

by ZEB1 knockdown (Fig. 6B). In addition, circCSPP1 knockdown induced G1 arrest, while circCSPP1 overexpression promoted G1 arrest in colon cancer cells (Additional file 3: Fig. S3A and B). The potential mechanism through which circCSPP1 regulates the progression of colon cancer are presented in Fig. 6C. The schematic diagram illustrates that circCSPP1 promotes the progression of colon cancer by regulating the miR-431/ROCK1 and miR-431/ZEB1 pathways.

\section{Discussion}

Increasing evidence has uncovered the critical role of circRNAs in the progression of human cancers, including colon cancer $[19,20]$. Various studies have reported that
circRNAs including circCCDC66 [27], circRNA_100859 [28] and circPPP1R12A [29] were upregulated in colon cancer and high expression was associated with a poor prognosis of the patients. In the present study, the dysregulated circRNAs were analyzed using the GEO database. A novel circRNA back-splicing 8-11 exons of the CSPP1 gene, termed circCSPP1, was found, which plays a tumor-promoting role in colon cancer.

Subsequently, the mechanisms of circCSPP1 regulating the progression of colon cancer were explored. The upregulated expression and the stability of circCSPP1 render it a potential biomarker and a diagnostic and therapeutic target in human cancers. CircCSPP1 was previous to be upregulated in liver cancer [30], prostate 
A

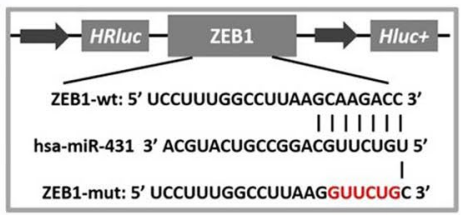

B

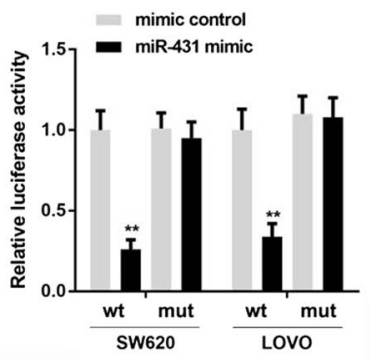

E

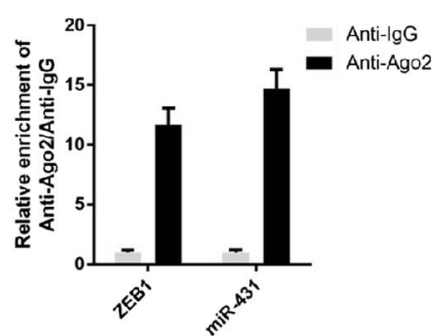

C

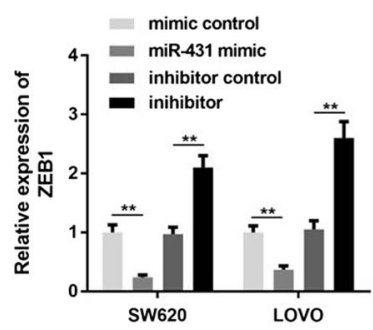

$\mathbf{F}$

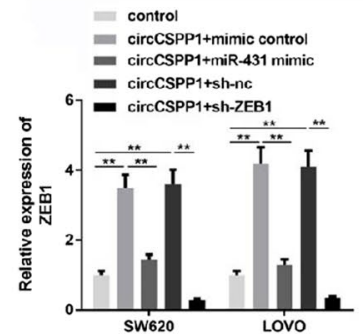

G

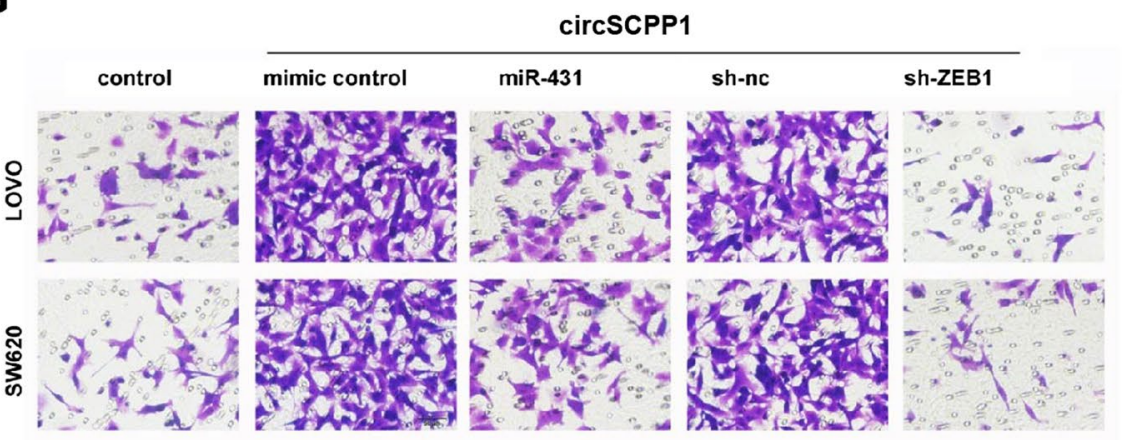

= control

- circCSPP1+mimic control

- circCSPP1+miR-431 miric

- circCSPP $1+$ sh-nc

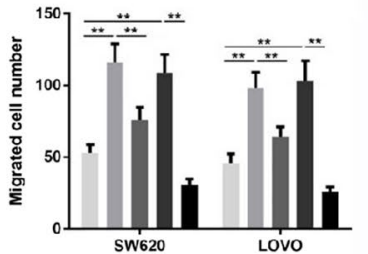

- control

- circCSPP1+mimic control

= circCSPP1+miR-431 mimic

aircCSPP1+sh-nc

circCSPP1+sh-ZEB1
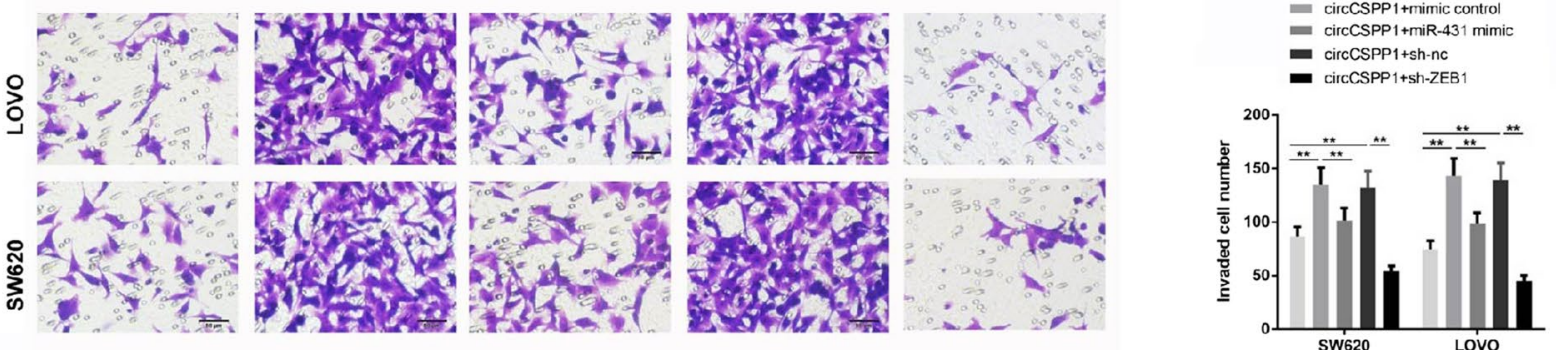

Fig. 5 MiR-431 targets ZEB1 in colon cancer cells. A The binding site between miR-431 and ROCK1 was predicted. B Luciferase reporter experiment was performed to detect the interaction between miR-431 and ZEB1. C and D colon cancer cells were treated with miR-431 mimics or miR-431 inhibitor for $24 \mathrm{~h}$, the gene and protein level of ZEB1 was detected with RT-qPCR and WB, respectively. E RIP assay was performed to verify the binding between ZEB1 and miR-431. Colon cancer cells were treated with pcDNA3.1-circCSPP1, pcDNA3.1-circCSPP1 plus miR-431 mimics or pcDNA3.1-circCSPP1 plus ZEB1 shRNA. F. RT-qPCR was used to detect the expression of ZEB1. G and $\mathbf{H}$ Transwell assay was performed to assess the migration and invasion of colon cancer cells. ${ }^{* *} p<0.01 ; n=3$

cancer [31], ovarian cancer [32] and glioma cancer [33]. In addition, circCSPP1 sponges different miRNAs including miR-520 h, miR-378 and miR-1182 to regulate tumor progression in these studies. The possible reason is that these miRNAs combine different specific 3'untranslated regions of circCSPP1. These may indicate circCSPP1 is 

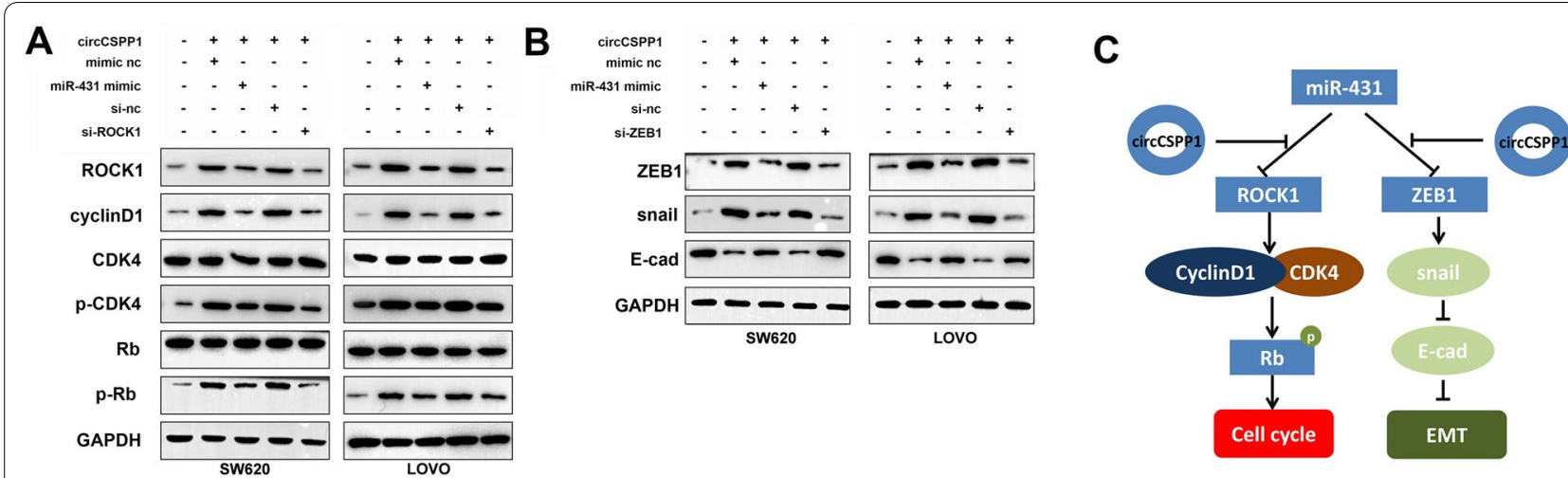

Fig. 6 CircCSPP1 upregulates cyclin D1/CDK4/RB signaling pathway in colon cancer. Colon cancer cells were treated with pcDNA3.1-circCSPP1, pcDNA3.1-circCSPP1 plus miR-431 mimics or pCDNA3.1-circCSPP1 plus ROCK1 shRNA. A, B Western blot was used to detect the expressions of cell cycle-related proteins (cyclin D1, CDK4, Rb) and EMT related protein (Snail, E-cadherin) in each group. C The potential mechanism by which circCSPP1 regulated the progression of colon cancer was presented. $\mathrm{N}=3$

an active oncogenic factor. Similar to these reports, the present study found that circCSPP1 promoted the progression of colon cancer by sponging a new microRNA miR-431 and regulating the expression of ROCK1 and ZEB1.

ROCK1 and ROCK2 are Rho-GTPase effectors that control vital aspects of the actin cytoskeleton. The RhoA/ ROCK pathway is activated in a variety of tumors and exerts a direct regulatory effect on the mobility of tumor cells [34-36]. Previous research has indicated that G1/S progression requires ROCK [37]. The role of ROCK1 in the regulation of the cell cycle may explain its effect on the proliferation of colon cancer cells. Our findings demonstrated that the knockdown of ROCK1 reversed the tumor-promoting effects of circCSPP1. The other function of ROCK1 is to induce the expression of cyclindependent kinase inhibitor (CDKI) p16, which prevents the CDK4/6-mediated phosphorylation of $\mathrm{Rb}$ proteins, thereby blocking E2F-dependent transcription [38]. The present study found that circCSPP1 promoted the expression of cyclin D1, $\mathrm{p}-\mathrm{CDK} 4$ and $\mathrm{p}-\mathrm{Rb}$ through the regulation of ROCK1 and miR-431. In addition, ZEB1 is well-known to be involved in the regulation of EMT in cancer cells [39]. In the present study, we found that circCSPP1 promoted EMT in colon cancer by modulating ZEB1.

In addition, recently, many studies have reported that circRNAs may encode proteins or peptides to participate in tumor progression [40], such as cricAXIN1 [41], circMAPK14 [42] and circCUX1 [43]. However, we did not study the encoding ability of circCSPP1. The issue is interesting, and it will be the focus of our further research.

In conclusion, the findings of the present study demonstrated that circCSPP1 was upregulated in colon cancer and functioned as an oncogene. In addition, circCSPP1 promoted the progression of colon cancer functions as a competing endogenous RNA by the regulating miR-431/ ROCK1 and miR-431/ZEB1 pathways. The findings presented herein may provide novel insight into the pathogenesis of colon cancer. However, what factors regulate circCSPP1 to play a role in promoting cancer progression still need to be further studied. In addition, the potential translation function of circCSPP1 also needs further investigation.

\section{Supplementary Information}

The online version contains supplementary material available at https://doi. org/10.1186/s12967-022-03240-x.

Additional file 1: Figure S1.CircCSPP1 targets miR-431 in colon cancer cells. (A) Luciferase reporter assay was performed to screen out the candidate miRNAs binding with circCSPP1. (B) The potential binding sites between miR-431 and circCSPP1. (C) Luciferase reporter experiment verified the binding relationship between circCSPP1 and miR-431. (D) The localization of miR-431 and circCSPP1 in colon cancer cells were detected with FISH experiment. (E) RIP experiment was carried out to confirm the interaction between circCSPP1 and miR-431. (F) RNA pull down was used to verify the interaction of circCSPP1 and miR-431. ${ }^{* *} \mathrm{p}<0.01$ $\mathrm{n}=3$.Additional file 2: Figure S2. MiR-431 targets ROCK1 in colon cancer cells. (A) The potential targets of miR-431 were predicted and the enrichment analysis was detected with RT-qPCR. (B) RT-qPCR was performed to analyze the expression of ROCK1 in colon cancer and adjacent normal tissues. (C) The potential binding sites between miR-431 and ROCK1 was presented. (D) Luciferase reporter experiment verified the binding relationship between miR-431 and ROCK1. (E) RIP experiment was carried out to confirm the interaction between miR-431 and ROCK1. (F) RNA pull down was used to verify the interaction of miR-431 and ROCK1. ${ }^{* *} p<0.01$; $\mathrm{n}=3$.Additional file 3: Figure S3. CircCSPP1 regulates cell cycle in colon cancer. Colon cancer cells were treated with circCSPP1 shRNA2, shRNA-nc, pcDNA3.1-circCSPP1, or pcDNA3.1-control (scramble). (A, B) The cell cycle distribution was detected with PI staining method and flow cytometric analysis. ${ }^{* *} \mathrm{p}<0.01 ; \mathrm{n}=3$.Additional file 4: Figure S4. Cell transfection experiments. Colon cancer cells were treated with pcDNA3.1-circCSPP1, pcDNA3.1-control, shRNA-nc, ROCK1 shRNA, ZEB1 shRNA, miR-431 mimic or miR-431 inhibitor. (A-E) The gene expression of circCSPP1, ROCK1, ZEB1 and miR-431 in cells were detected with RT-qPCR. ${ }^{* *} p<0.01 ; n=3$. 


\section{Acknowledgements \\ Not applicable.}

\section{Authors' contributions}

JW, LZ, AW and XZ contributed to the study design. BC, ZY and JZ conducted the literature search. JW, BC and $Z Y$ acquired the data. JW and ZZ wrote the article. $L Z, C H$ and $Y B$ performed data analysis. YB, XR, SW and JO revised the article. AW and $X Z$ gave the final approval of the version to be submitted. All authors read and approved the final manuscript.

\section{Funding}

This study was supported by grants from the Youth Science and Technology Program, Science and Education Promoting Health Project of Suzhou City, China (no. KJXW2021086). National Science Foundation of China (NSFC, No. 31770985, No. 82073180). Jiangsu Provincial Key Research and Development Program, China (No. BE2019665). Jiangsu Provincial Medical Youth Talent, China (No. QNRC2016732). Suzhou Municipal Project of Gusu Health Talent, Young Top Talent, China (No. 2018-057). Gusu Health Talents Cultivation Program, China (No. GSWS2019028). Scientific Research Program of Jiangsu Provincial "333 Projects", China (No. BRA2019327). Science and Technology Program of Suzhou City, China (No. SYS2019053, No. SLC201906).

\section{Availability of data and materials}

The datasets used and/or analyzed during the current study are available from the corresponding author on reasonable request.

\section{Declarations}

Ethics approval and consent to participate

This study was approved by the First Affiliated Hospital of Soochow University Ethical Committee (Approval No. 2019834). All animal housing and experiments were conducted in strict accordance with the Institutional Guidelines for Care and Use of Laboratory Animals.

\section{Consent for publication}

Not applicable.

\section{Competing interests}

These authors declared no competing interests in this research.

\section{Author details}

${ }^{1}$ Department of General Surgery, The First Affiliated Hospital of Soochow University, Suzhou, China. ${ }^{2}$ Department of General Surgery, Suzhou Dushu Lake Hospital (Dushu Lake Hospital Affiliated to Soochow University), Suzhou, China. ${ }^{3}$ Jiangsu Institute of Clinical Immunology, The First Affiliated Hospital of Soochow University, Suzhou, China. ${ }^{4}$ Jiangsu Key Laboratory of Clinical Immunology, Soochow University, Suzhou, China. ${ }^{5}$ Jiangsu Key Laboratory of Gastrointestinal Tumor Immunology, Suzhou, China. ${ }^{6}$ Department of Gastroenterology, Suzhou Hospital of Integrated Traditional Chinese and Western Medicine, Suzhou, China. ${ }^{7}$ Department of Gastroenterology, The First Affiliated Hospital of Soochow University, Suzhou, China. ${ }^{8}$ Department of Urinary Surgery, The First Affiliated Hospital of Soochow University, Suzhou, China.

Received: 23 December 2021 Accepted: 8 January 2022

Published online: 31 January 2022

\section{References}

1. Zhang J, Liu H, Zhao P, Zhou H, Mao T. Has_circ_0055625 from circRNA profile increases colon cancer cell growth by sponging miR-106b-5p. J Cell Biochem. 2019;120(3):3027-37.

2. Wang L, Peng X, Lu X, Wei Q, Chen M, Liu L. Inhibition of hsa circ_0001313 (circCCDC66) induction enhances the radio-sensitivity of colon cancer cells via tumor suppressor miR-338-3p: effects of cicr_0001313 on colon cancer radio-sensitivity. Pathol Res Pract. 2019;215(4):689-96.

3. Feng J, Ma J, Liu S, Wang J, Chen Y. A noncoding RNA LINC00504 interacts with c-Myc to regulate tumor metabolism in colon cancer. J Cell Biochem. 2019. https://doi.org/10.1002/jcb.28733.
4. Zhang X, Liu H, Sun B, Sun Y, Zhong W, Liu Y, Chen S, Ling H, Zhou L, Jing $X$, et al. Novel podophyllotoxin derivatives as partial PPARgamma agonists and their effects on insulin resistance and type 2 diabetes. Sci Rep. 2016;6:37323.

5. Miller KD, Nogueira L, Mariotto AB, Rowland JH, Yabroff KR, Alfano CM Jemal A, Kramer JL, Siegel RL. Cancer treatment and survivorship statistics, 2019. CA A Cancer J Clin. 2019;69(5):363-85.

6. Miller KD, Nogueira L, Mariotto AB, Rowland JH. Clinicians RLSJCACJf: cancer treatment and survivorship statistics, 2019. CA A Cancer J Clin. 2019. https://doi.org/10.3322/caac.21565.

7. Zhong W, Chen S, Zhang Q, Xiao T, Qin Y, Gu J, Sun B, Liu Y, Jing X, Hu X, et al. Doxycycline directly targets PAR1 to suppress tumor progression. Oncotarget. 2017:8(10):16829-42.

8. Yang S, Zhang H, Guo L, Zhao Y, Chen F. Reconstructing the coding and non-coding RNA regulatory networks of miRNAs and mRNAs in breast cancer. Gene. 2014;548(1):6-13.

9. Siciliano V, Garzilli I, Fracassi C, Criscuolo S, Ventre S, di Bernardo D. MiRNAs confer phenotypic robustness to gene networks by suppressing biological noise. Nat Commun. 2013;4:2364.

10. Zhang B, Pan X, Cobb GP, Anderson TA. microRNAs as oncogenes and tumor suppressors. Dev Biol. 2007;302(1):1-12.

11. Liu B, Shyr Y, Cai J, Liu Q. Interplay between miRNAs and host genes and their role in cancer. Brief Funct Genomics. 2018;18(4):255-66.

12. Chen Q, Liu T, Bao Y, Zhao T, Wang J, Wang H, Wang A, Gan X, Wu Z, Wang L. CircRNA CRAPGEF5 inhibits the growth and metastasis of renal cell carcinoma via the miR-27a-3p/TXNIP pathway. Cancer Lett. 2020;469:68-77.

13. Wei S, Zheng Y, Jiang Y, Li X, Geng J, Shen Y, Li Q, Wang X, Zhao C, Chen $Y$, et al. The circRNA circPTPRA suppresses epithelial-mesenchymal transitioning and metastasis of NSCLC cells by sponging miR-96-5p. EBioMedicine. 2019;44:182-93.

14. Song T, Xu A, Zhang Z, Gao F, Zhao L, Chen X, Gao J, Kong X. CircRNA hsa_circRNA_101996 increases cervical cancer proliferation and invasion through activating TPX2 expression by restraining miR-8075. J Cell Physiol. 2019;234(8):14296-305.

15. Zhong W, Liu P, Zhang Q, Li D, Lin J. Structure-based QSAR, molecule design and bioassays of protease-activated receptor 1 inhibitors. J Biomol Struct Dyn. 2017;35(13):2853-67.

16. Zhao J, Xia H, Wu Y, Lu L, Cheng C, Sun J, Xiang Q, Bian T, Liu Q. CircRNA_0026344 via miR-21 is involved in cigarette smoke-induced autophagy and apoptosis of alveolar epithelial cells in emphysema. Cell Biol Toxicol. 2021. https://doi.org/10.1007/s10565-021-09654-5.

17. Guo Z, Xie M, Zou Y, Liang Q, Liu F, Su J, He Z, Cai X, Chen Z, Zhao Q, et al. Circular RNA Hsa_circ_0006766 targets microRNA miR-4739 to regulate osteogenic differentiation of human bone marrow mesenchymal stem cells. Bioengineered. 2021;12(1):5679-87.

18. Li X, Ding J, Wang X, Cheng Z, Zhu Q. NUDT21 regulates circRNA cyclization and ceRNA crosstalk in hepatocellular carcinoma. Oncogene. 2019. https://doi.org/10.1038/s41388-019-1030-0.

19. Verduci L, Strano S, Yarden Y, Blandino G. The circRNA-microRNA code: emerging implications for cancer diagnosis and treatment. Mol Oncol. 2019;13(4):669-80.

20. Yu J, Yang M, Zhou B, Luo J, Zhang Z, Zhang W, Yan Z. CircRNA-104718 acts as competing endogenous RNA and promotes hepatocellular carcinoma progression through microRNA-218-5p/TXNDC5 signaling pathway. Clin Sci. 2019;133(13):1487-503.

21. Livak KJ, Schmittgen TD. Analysis of relative gene expression data using real-time quantitative PCR and the 2(-Delta Delta $C(T)$ ) method. Methods. 2001;25(4):402-8

22. Meng J, Ai X, Lei Y, Zhong W, Qian B, Qiao K, Wang X, Zhou B, Wang H, Huai L, et al. USP5 promotes epithelial-mesenchymal transition by stabilizing SLUG in hepatocellular carcinoma. Theranostics. 2019;9(2):573-87.

23. Zhong W, Yang W, Qin Y, Gu W, Xue Y, Tang Y, Xu H, Wang H, Zhang C, Wang C, et al. 6-Gingerol stabilized the p-VEGFR2/VE-cadherin/betacatenin/actin complex promotes microvessel normalization and suppresses tumor progression. J Exp Clin Cancer Res. 2019;38(1):285.

24. Zheng $X$, Huang $M$, Xing $L$, Yang $R$, Wang $X$, Jiang $R$, Zhang $L$, Chen J. The circRNA circSEPT9 mediated by E2F1 and EIF4A3 facilitates the carcinogenesis and development of triple-negative breast cancer. Mol Cancer. 2020;19(1):73. 
25. Zhong W, Sun B, Gao W, Qin Y, Zhang H, Huai L, Tang Y, Liang Y, He L, Zhang $X$, et al. Salvianolic acid A targeting the transgelin-actin complex to enhance vasoconstriction. EBioMedicine. 2018;37:246-58.

26. Wang Y, Sun J, Ma C, Gao W, Song B, Xue H, Chen W, Chen X, Zhang Y, Shao $Q$, et al. Reduced expression of galectin-9 contributes to a poor outcome in colon cancer by inhibiting NK cell chemotaxis partially through the Rho/ROCK1 signaling pathway. PloS ONE. 2016;1 1(3): e0152599.

27. Hsiao KY, Lin YC, Gupta SK, Chang N, Yen L, Sun HS, Tsai SJ. Noncoding effects of circular RNA CCDC66 promote colon cancer growth and metastasis. Can Res. 2017;77(9):2339-50.

28. Zhou P, Xie W, Huang HL, Huang RQ, Tian C, Zhu HB, Dai YH, Li ZY. circRNA_100859 functions as an oncogene in colon cancer by sponging the miR-217-HIF-1alpha pathway. Aging. 2020;12(13):13338-53.

29. Zheng X, Chen L, Zhou Y, Wang Q, Zheng Z, Xu B, Wu C, Zhou Q, Hu W, Wu C, et al. A novel protein encoded by a circular RNA circPPP1R12A promotes tumor pathogenesis and metastasis of colon cancer via Hippo-YAP signaling. Mol Cancer. 2019;18(1):47.

30. Jia N, Song Z, Chen B, Cheng J, Zhou W. A novel circular RNA circCSPP1 promotes liver cancer progression by sponging miR-1182. Onco Targets Ther. 2021;14:2829-38.

31. Lu J, Zhong C, Luo J, Shu F, Lv D, Liu Z, Tan X, Wang S, Wu K, Yang T, et al. HnRNP-L-regulated circCSPP1/miR-520h/EGR1 axis modulates autophagy and promotes progression in prostate cancer. Mol Ther Nucleic Acids. 2021;26:927-44.

32. Li QH, Liu Y, Chen S, Zong ZH, Du YP, Sheng XJ, Zhao Y. circ-CSPP1 promotes proliferation, invasion and migration of ovarian cancer cells by acting as a miR-1236-3p sponge. Biomed Pharmacother. 2019;114: 108832.

33. Xue YF, Li M, Li W, Lin Q, Yu BX, Zhu QB, Chen HJ. Roles of circ-CSPP1 on the proliferation and metastasis of glioma cancer. Eur Rev Med Pharmacol Sci. 2020;24(10):5519-25.

34. Huang GX, Wang Y, Su J, Zhou P, Li B, Yin LJ, Lu J. Up-regulation of Rhoassociated kinase $1 / 2$ by glucocorticoids promotes migration, invasion and metastasis of melanoma. Cancer Lett. 2017:410:1-11.

35. Tang Y, He Y, Zhang P, Wang J, Fan C, Yang L, Xiong F, Zhang S, Gong Z, Nie $\mathrm{S}$, et al. LncRNAs regulate the cytoskeleton and related Rho/ROCK signaling in cancer metastasis. Mol Cancer. 2018;17(1):77.

36. Olson MF. Contraction reaction: mechanical regulation of Rho GTPase. Trends Cell Biol. 2004;14(3):111-4.

37. Kim H, Kang M, Lee SA, Kwak TK, Jung O, Lee HJ, Kim SH, Lee JW. TM4SF5 accelerates G1/S phase progression via cytosolic p27Kip1 expression and RhoA activity. Biochim Biophys Acta. 2010;1803(8):975-82.

38. Luh FY, Archer SJ, Domaille PJ, Smith BO, Owen D, Brotherton DH, Raine AR, Xu X, Brizuela L, Brenner SL, et al. Structure of the cyclin-dependent kinase inhibitor p19Ink4d. Nature. 1997;389(6654):999-1003.

39. Lee SY, Jeong EK, Ju MK, Jeon HM, Kim MY, Kim CH, Park HG, Han SI, Kang HS. Induction of metastasis, cancer stem cell phenotype, and oncogenic metabolism in cancer cells by ionizing radiation. Mol Cancer. 2017;16(1):10

40. Qian L, Yu S, Chen Z, Meng Z, Huang S, Wang P. The emerging role of circRNAs and their clinical significance in human cancers. Biochim Biophys Acta Rev Cancer. 2018;1870(2):247-60.

41. Peng Y, Xu Y, Zhang X, Deng S, Yuan Y, Luo X, Hossain MT, Zhu X, Du K, Hu $F$, et al. A novel protein AXIN1-295aa encoded by circAXIN1 activates the Wnt/beta-catenin signaling pathway to promote gastric cancer progression. Mol Cancer. 2021;20(1):158.

42. Wang L, Zhou J, Zhang C, Chen R, Sun Q, Yang P, Peng C, Tan Y, Jin C, Wang $T$, et al. A novel tumour suppressor protein encoded by circMAPK14 inhibits progression and metastasis of colorectal cancer by competitively binding to MKK6. Clin TransI Med. 2021;11(10): e613.

43. Yang F, Hu A, Guo Y, Wang J, Li D, Wang X, Jin S, Yuan B, Cai S, Zhou Y, et al. p113 isoform encoded by CUX1 circular RNA drives tumor progression via facilitating ZRF1/BRD4 transactivation. Mol Cancer. 2021;20(1):123.

\section{Publisher's Note}

Springer Nature remains neutral with regard to jurisdictional claims in published maps and institutional affiliations.

Ready to submit your research? Choose BMC and benefit from:

- fast, convenient online submission

- thorough peer review by experienced researchers in your field

- rapid publication on acceptance

- support for research data, including large and complex data types

- gold Open Access which fosters wider collaboration and increased citations

- maximum visibility for your research: over 100M website views per year

At BMC, research is always in progress.

Learn more biomedcentral.com/submissions 